\title{
Determination of the Distal Fusion Level in the Management of Thoracolumbar and Lumbar Adolescent Idiopathic Scoliosis Using Pedicle Screw Instrumentation
}

\author{
Sung-Soo Kim ${ }^{1}$, Dong-Ju Lim² ${ }^{2}$ Jin-Hyok Kim², Jong-Woo Kim², \\ Kyu-Sub Um², Soo-Hyung Ahn ${ }^{2}$, Se-Il Suk ${ }^{2}$ \\ ${ }^{1}$ Department of Orthopaedics, Haeundae Paik Hospital, Inje University College of Medicine, Busan, Korea \\ ${ }^{2}$ Seoul Spine Institute, Sanggye Paik Hospital, Inje University College of Medicine, Seoul, Korea
}

\section{Study Design: A retrospective study.}

Purpose: To determine the exact distal fusion level in the management of thoracolumbar/lumbar adolescent idiopathic scoliosis (TL/L AIS) using pedicle screw instrumentation (PSI).

Overview of Literature: The selection of distal fusion level remains controversial in TL/L AIS.

Methods: Radiographic parameters of 66 TL/L AIS patients were analyzed. The patients were grouped according to the distal fusion level; $L 3$ group (fusion to $L 3, n=58$ ) and $L 4$ group (fusion to $L 4, n=8$ ). The $L 3$ group was subdivided into $L 3 A$ ( $L 3$ crosses the mid-sacral line with rotation of less than grade II, $n=33$ ) and $L 3 B$ ( $L 3$ does not cross the mid-sacral line or rotation is grade $\|$ or more, $n=25$ ) based on both bending radiographs. All of the patients in the $L 4$ group had the same location and rotation of $L 3$ in bending films as that of patients in the L3B group. An unsatisfactory result was defined as a lowest instrumented vertebral tilt (LIVT) of more than $10^{\circ}$ or coronal balance of more than $15 \mathrm{~mm}$.

Results: Among the 3 groups, there was a significantly lesser correction in the TL/L curve and LIVT in the L3B group. Unsatisfactory results were obtained in 3 patients (9.1\%) of the L3A group, in 15 patients (68.2\%) of the L3B group, and in 1 patient (12.5\%) of the L4 group with a significant difference.

Conclusions: In TL/L AIS treatment with PSI, the curve can be fused to L3 with favorable radiographic outcomes when L3 crosses the mid-sacral line with rotation of less than grade II in bending films. Otherwise, fusion has to be extended to L4.

Keywords: Scoliosis; Adolescent; Spinal fusion; Instrumentation

\section{Introduction}

The ideal surgical treatment for adolescent idiopathic scoliosis (AIS) should produce correction of the three- dimensional deformity with a stable and balanced spine and preserve as many distal motion segments as possible. Although there are a fewer number of patients with thoracolumbar/lumbar (TL/L) AIS than those with thoracic

Received May 2, 2014; Revised May 15, 2014; Accepted May 15, 2014

Corresponding author: Dong-Ju Lim

Seoul Spine Institute, Sanggye-Paik Hospital, Inje University College of Medicine,

1342 Dongil-ro, Nowon-gu, Seoul 139-707, Korea

Tel: +82-2-950-1288, Fax: +82-2-934-6342, E-mail: scd25@paik.ac.kr 
AIS [1], spine surgeons treating these conditions encounter some difficulties, such as how to correct a C-shaped curve in the coronal plane into an S-shape curve in the sagittal plane and which vertebra should be selected as the lowest instrumented vertebra (LIV).

Anterior spinal fusion for TL/L AIS offers relatively shorter fusion with greater correction compared to posterior spinal fusion [2-5]. It is also associated with mechanical failure of fixation devices, a high rate of pseudarthrosis, loss of correction and kyphosis in the instrumented segment $[5,6]$. Although these complications have been reduced to a certain degree with the development of rigid instrumentation, there is a risk of major vessel or internal organ injury in anterior surgery and most spine surgeons are not familiar with this technique.

It was accepted in the late 1950s that posterior spinal fusion with Harrington instrumentation could be successfully used for AIS. In this method, the LIV was the stable vertebra. In spite of achieving considerable coronal plane correction, it caused flattening of the sagittal contour with long segment fusion and distal adjacent segment degeneration [7-13].

After segmental pedicle screw instrumentation (PSI) was introduced for the correction of scoliosis, it provide an enhanced three-dimensional deformity correction and preservation of the motion segments by reducing the number of levels fused [14-17]. For performing this powerful instrumentation, either L3 or L4 has been commonly chosen as the LIV for TL/L AIS.

There is still some controversy on whether to fuse the TL/L curve to L3 or L4. This article retrospectively analyzed the surgical outcomes of the patients with TL/L AIS treated by PSI and determined the exact distal fusion level.

\section{Materials and Methods}

This study retrospectively reviewed 66 patients with TL/ L AIS (22 thoracolumbar and 44 lumbar; lenke 5) treated using posterior PSI with a minimum follow-up of 2 years (range, 2.1-10.7 years). The mean age at the time of surgery was 15.2 years (range, 10.7-17.8 years). There were 9 males and 57 females and the male to female ratio was 1:6.3. Left convex curve was found in 56 patients, and right convex curve was found in 10 patients with the caudal end vertebra of L3 or L4.

The spine was evaluated by preoperative and postop- erative standing anteroposterior and lateral radiographs to determine curve magnitude, sagittal alignment, lower instrumented vertebral tilt (LIVT), and coronal balance. The curve magnitude was measured using the Cobb method. Sagittal alignment was checked by assessing thoracic kyphosis and lumbar lordosis. Thoracic kyphosis was measured by the Cobb method from the superior endplate of $\mathrm{T} 5$ to the inferior endplate of T12, and lumbar lordosis was measured from the superior endplate of T12 to the endplate of S1. The LIVT was calculated as the angle between the inferior endplate of the LIV and a horizontal line. The coronal balance was measured as the distance between the $\mathrm{C} 7$ plumb line and the central sacral vertical line (CSVL). A LIVT of more than $10^{\circ}$ or a coronal balance of more than $15 \mathrm{~mm}$ was considered unsatisfactory.

The preoperative bending radiographs were taken to evaluate the location and rotation of L3. The bending radiograph was to be obtained during maximal active right and left bending in the supine position by the patient, and not during passive bending by the examiner.

The patients were grouped according to the level of the LIV for comparison of radiographic parameters. The L3 group included 58 patients having L3 as the LIV, and the L4 group included 8 patients having L4 as the LIV. The L3 group was subdivided into two subgroups based on the location and rotation of $\mathrm{L} 3$ in the preoperative bending radiographs. In the L3A group, L3 crossed the midsacral line in the film taken during bending towards the concave side (for example, right bending film in the left curve in Fig. 1C) and rotated less than Nash-Moe grade II in the film during bending towards the convex side (left bending film in the left curve in Fig. 1D). The L3B group was defined when L3 did not cross the mid-sacral line in right bending or had rotation of grade II or more in left bending. Therefore, the patients who had at least one of these two radiographic parameters were grouped into the L3B group (Fig. 2C, D). There were 33 patients in the L3A group and 25 patients in the L3B group. In all of the patients in the L4 group, L3 did not cross the mid-sacral line nor had rotation of grade II or more in bending films, similar to that in the L3B group.

Statistical analysis was performed using MedCalc for Windows, ver. 13.1.2.0 (MedCalc Software, Ostend, Belgium). The independent $t$-test and the Mann-Whitney test were used for performing parametric and nonparametric comparison of the two groups, respectively, and 

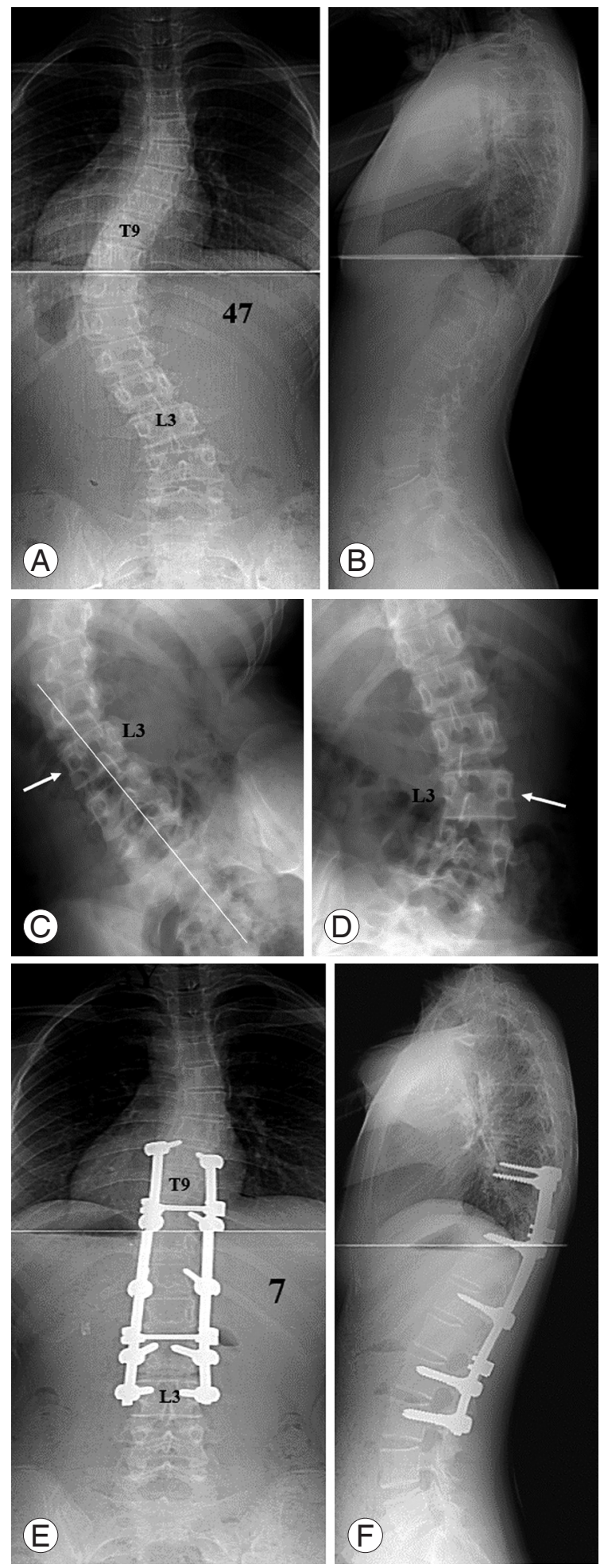

Fig. 1. (A, B) Preoperative standing anteroposterior and lateral radiographs of a 13.3 -year-old girl with $47^{\circ}$ thoracolumbar curve. (C) L3 (arrow) crossed the mid-sacral line in the right bending radiograph. (D) L3 (arrow) rotated less than Nash-Moe grade II, and it actually rotated in the opposite direction in the left bending radiograph. (E, F) Anteroposterior and lateral radiographs taken at 3.5 years after operation. The fusion was extended down to $L 3$. The thoracolumbar curve was corrected to $7^{\circ}$ with well-balanced spine and horizontalization of the lowest instrumented vertebra.
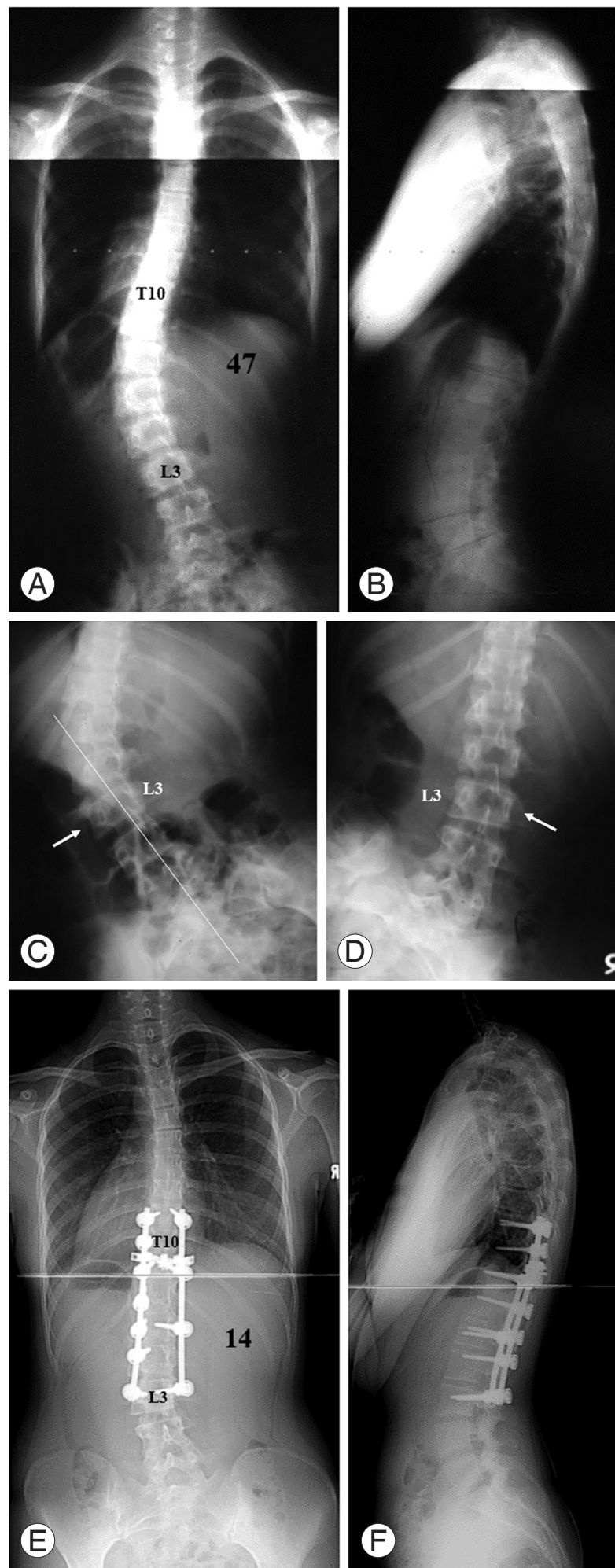

Fig. 2. (A, B) Preoperative standing anteroposterior and lateral radiographs of a 14.1 -year-old girl with $47^{\circ}$ thoracolumbar curve. (C) L3 (arrow) did not cross the mid-sacral line in the right bending radiograph. (D) L3 (arrow) rotated less than Nash-Moe grade II in the left bending radiograph. $(\mathbf{E}, \mathbf{F})$ Anteroposterior and lateral radiographs taken at 4.5 years after operation. The fusion was extended down to $L 3$. The thoracolumbar curve was corrected to $20^{\circ}$ with a lower instrumented vertebra tilt of $15^{\circ}$ and coronal imbalance. 
the Kruskal-Wallis test was chosen for comparing the three groups. The preoperative and postoperative values in each group were analyzed with the paired $t$-test for performing parametric comparison and the Wilcoxon test for performing nonparametric comparison. The percentage of unsatisfactory results in one group was compared with that in another group using the Fisher's exact test. A $p$-value of less than 0.05 was considered statistically significant.

\section{Surgical techniques}

All of the surgeries were performed via the standard posterior approach using PSI. The deformities were treated by performing TL/L fusion, usually extending from the proximal neutral vertebra or one level proximal to the upper end vertebra to L3 or L4. The surgical incision spanned from the spinous process two levels above the uppermost instrumented vertebra to the lamina of the LIV. After posterior exposure, facets included in the fusion were destroyed by inferior facetectomy and removal of the articular cartilage was performed to promote intraarticular fusion. The presumed pedicle entry points (PPEP) were decorticated with a rongeur or burr to facilitate the insertion of guide pins. The PPEP of the lower thoracic spine and lumbar spine was at the junction of the bisected transverse process and the lateral margin of the facet joint. Then guide pins were inserted at a depth of about $1 \mathrm{~cm}$ through the exposed cancellous bone at the PPEP. On the intraoperative posteroanterior and lateral radiographs, the ideal pedicle entry point (IPEP) and the direction of the screw were determined. The IPEP in a neutrally rotated vertebra was at the junction of a line parallel to the vertebral end plates bisecting the pedicle and the lateral margin of the pedicle ring shadow on the posteroanterior film, and the ideal direction was parallel to the vertebral endplates on the lateral film. In the rotated vertebra, the IPEP on the convex side moved more medially, whereas the IPEP on the opposite side (concave side) moved more laterally with increasing vertebral rotation.

After determining the position of the IPEP and the direction of the ideal pedicle path relative to the guide pin, the pedicle was entered with an awl and then a smalldiameter drill or curette. The hole was checked with a blunt-ended probe. A safe entry into the pedicle was confirmed when the probe meet bony resistance in all directions. Pedicle screw was turned with gentle force so that the screw followed the predrilled path. Pedicle screws were inserted in every vertebra on the convex side of the TL/L curve (correction side) and in every second or third vertebra on the concave side of the TL/L curve (supporting side). In the large or rigid curve, pedicle screws were inserted segmentally on the concave side to improve deformity correction. After inserting the screws, a rod contoured to have a slight exaggeration of the normal sagittal alignment in the instrumented zone, which is S-shaped in the TL spine, was inserted into the correction side. Insertion of the rod was difficult when there was a large discrepancy between the C-shaped contour of the vertebral column due to the TL/L coronal curve and the S-shape of the rod. This was facilitated by using rod introducers or sequentially closing the screw caps while rotating the rod to fit the contour of the vertebral column. Long arm reduction screws can be used effectively and both rods can be manipulated simultaneously as well. The correction of the deformity was performed by rod derotation and translation. When considerable straightening of the contoured rod occurred during the process of deformity correction, the rod was removed. Then, the removed rod or another new rod was recontoured, and the same procedure was performed to achieve better correction of both coronal and sagittal plane deformities. After locking the rod on the correction side, the opposite rod was bent to conform to the shape of the corrected curve and was placed on the supporting side in situ. The two rods were connected by means of a transverse connector. Subsequently, posterior fusion was performed.

\section{Results}

\section{Deformity correction}

When taken as a whole, the preoperative major TL/L curve measuring $54.1^{\circ} \pm 6.6^{\circ}$ was reduced to $14.5^{\circ} \pm 7.3^{\circ}$ at the final follow-up, thus showing a $74.3 \%$ correction. The preoperative thoracolumbar curve measuring $52.8^{\circ} \pm 6.1^{\circ}$ and lumbar curve measuring $54.7^{\circ} \pm 6.8^{\circ}$ were reduced to $14.3^{\circ} \pm 5.4^{\circ}$ and $14.4^{\circ} \pm 8.2^{\circ}$ at the final follow-up with a curve correction of $72.3 \%$ and $75.3 \%$, respectively. On comparing the three groups, the preoperative TL/L curve was reduced from $53.8^{\circ} \pm 5.4^{\circ}$ to $10.3^{\circ} \pm 3.4^{\circ}$ (80.9\% correction) in the L3A group, from $54.5^{\circ} \pm 8.0^{\circ}$ to $20.6^{\circ} \pm 7.5^{\circ}$ (63.5\% correction) in the L3B group, and from $56.2^{\circ} \pm 6.1^{\circ}$ to $11.4^{\circ} \pm 7.6^{\circ}(79.5 \%$ correction) in the L4 group (Table 1 ). 
Table 1. Preoperative and postoperative curve characteristics and unsatisfactory results

\begin{tabular}{|c|c|c|c|c|}
\hline & L3A & $\mathrm{L} 3 \mathrm{~B}$ & $\mathrm{~L} 4$ & Total \\
\hline No. of patients & 33 & 25 & 8 & 66 \\
\hline \multicolumn{5}{|l|}{$\mathrm{TL} / \mathrm{L}$ (major) curve $\left({ }^{\circ}\right.$ ) } \\
\hline Preoperative & $53.8 \pm 5.4$ & $54.5 \pm 8.0$ & $56.2 \pm 6.1$ & $54.1 \pm 6.6$ \\
\hline Postoperative & $10.3 \pm 3.4$ & $20.6 \pm 7.5$ & $11.4 \pm 7.6$ & $14.5 \pm 7.3$ \\
\hline Correction rate $(\%)$ & 80.9 & 63.5 & 79.5 & 74.3 \\
\hline \multicolumn{5}{|l|}{ Thoracic (minor) curve $\left({ }^{\circ}\right)$} \\
\hline Preoperative & $25.6 \pm 5.0$ & $26.7 \pm 5.5$ & $27.5 \pm 4.4$ & $26.4 \pm 5.2$ \\
\hline Postoperative & $14.9 \pm 4.3$ & $15.3 \pm 4.8$ & $15.8 \pm 3.7$ & $15.1 \pm 4.6$ \\
\hline Correction rate $(\%)$ & 43.9 & 42.4 & 44.2 & 43.3 \\
\hline \multicolumn{5}{|l|}{ Thoracic kyphosis $\left({ }^{\circ}\right)$} \\
\hline Preoperative & $20.2 \pm 8.5$ & $18.6 \pm 9.0$ & $21.3 \pm 6.4$ & $20.1 \pm 7.8$ \\
\hline Postoperative & $26.8 \pm 8.1$ & $23.6 \pm 9.1$ & $25.5 \pm 7.2$ & $25.7 \pm 8.2$ \\
\hline \multicolumn{5}{|l|}{ Lumbar lordosis $\left({ }^{\circ}\right)$} \\
\hline Preoperative & $50.0 \pm 9.1$ & $48.2 \pm 9.7$ & $47.7 \pm 8.2$ & $49.2 \pm 9.3$ \\
\hline Postoperative & $52.8 \pm 8.2$ & $51.7 \pm 10.3$ & $50.2 \pm 7.5$ & $52.1 \pm 8.9$ \\
\hline \multicolumn{5}{|l|}{$\operatorname{LIVT}\left({ }^{\circ}\right)$} \\
\hline Preoperative & $21.7 \pm 7.5$ & $23.5 \pm 7.3$ & $29.3 \pm 8.0$ & $23.2 \pm 7.8$ \\
\hline Postoperative & $3.3 \pm 2.9$ & $11.0 \pm 5.3$ & $5.5 \pm 5.8$ & $6.4 \pm 5.4$ \\
\hline Correction rate $(\%)$ & 84.3 & 53.4 & 81.5 & 72.6 \\
\hline \multicolumn{5}{|l|}{ Coronal balance (mm) } \\
\hline Preoperative & $14.6 \pm 8.2$ & $18.1 \pm 11.3$ & $16.4 \pm 12.2$ & $16.3 \pm 10.1$ \\
\hline Postoperative & $5.8 \pm 4.6$ & $12.2 \pm 9.1$ & $9.3 \pm 4.5$ & $8.4 \pm 7.2$ \\
\hline Unsatisfactory results (\%) & $3 / 33(9.1)$ & $15 / 25(68.2)$ & $1 / 8(12.5)$ & 19/66 (28.8) \\
\hline
\end{tabular}

TL/L, thoracolumbar/lumbar; LIVT, lower instrumented vertebral tilt.

Major curve correction was not significantly affected by the curve type (thoracolumbar vs. lumbar) and the LIV (L3 group vs. L4 group) ( $p>0.05)$. However, major curve correction was significantly less in the L3B group than the other two groups $(p<0.05)$.

In total, the preoperative thoracic Cobb angle measuring $26.4^{\circ} \pm 5.2^{\circ}$ spontaneously reduced to $15.1^{\circ} \pm 4.6^{\circ}$ at the final follow-up, demonstrating a $43.3 \%$ correction without instrumentation. In each group analysis, the preoperative thoracic Cobb angle reduced from $25.6^{\circ} \pm 5.0^{\circ}$ to $14.9^{\circ} \pm 4.3^{\circ}$ ( $43.9 \%$ correction) in the L3A group, from $26.7^{\circ} \pm 5.5^{\circ}$ to $15.3^{\circ} \pm 4.8^{\circ}(42.4 \%$ correction $)$ in the L3B group, and from $27.5^{\circ} \pm 4.4^{\circ}$ to $15.8^{\circ} \pm 3.7^{\circ}$ ( $44.2 \%$ correction) in the L4 group (Table 1). There was no significant difference in thoracic curve correction among the three groups.

The preoperative thoracic kyphosis measuring $20.1^{\circ} \pm 7.8^{\circ}$ and lumbar lordosis measuring $49.2^{\circ} \pm 9.3^{\circ}$ were changed to $25.7^{\circ} \pm 8.2^{\circ}$ and $52.1^{\circ} \pm 8.9^{\circ}$, respectively. Thoracic kyphosis was significantly increased after surgery compared with its preoperative value. Lumbar lordosis did not change significantly but it was maintained after surgery. Thoracolumbar kyphosis or significantly reduced lumbar lordosis was not detected during the follow-up. Sagittal parameters in each group are shown in Table 1. There was no significant difference in thoracic kyphosis and lumbar lordosis at the final follow-up among the three groups.

\section{LIVT and coronal balance}

The LIVT was reduced from $21.7^{\circ} \pm 7.5^{\circ}$ to $3.3^{\circ} \pm 2.9^{\circ}$ $\left(84.3 \%\right.$ correction) in the $\mathrm{L} 3 \mathrm{~A}$ group, from $23.5^{\circ} \pm 7.3^{\circ}$ to $11.0^{\circ} \pm 5.3^{\circ}(53.4 \%$ correction) in the L3B group, and from $29.3^{\circ} \pm 8.0^{\circ}$ to $5.5^{\circ} \pm 5.8^{\circ}(81.5 \%$ correction $)$ in the L4 
group. Among the three groups, there was a significantly less improvement in LIVT in the L3B group $(p<0.05)$. The coronal balance improved from $14.6 \pm 8.2$ to $5.8 \pm 4.6$ $\mathrm{mm}$ in the L3A group, from $18.1 \pm 11.3$ to $12.2 \pm 9.1 \mathrm{~mm}$ in the L3B group, and from $16.4 \pm 12.2$ to $9.3 \pm 4.5 \mathrm{~mm}$ in the L4 group (Table 1).

\section{Unsatisfactory results}

In this study, unsatisfactory results defined as a LIVT of more than $10^{\circ}$ or a coronal balance of more than 15 $\mathrm{mm}$ were obtained in 19 patients; 3 patients $(3 / 33,9.1 \%)$ in the L3A group, 15 patients $(15 / 25,68.2 \%)$ in the L3B group, and 1 patient $(1 / 8,12.5 \%)$ in the L4 group (Table 1). There was no significant difference in the percentage of postoperative unsatisfactory results between the L3A and L4 groups $(p>0.05)$, but there was a significant difference in the percentage of postoperative unsatisfactory results between the L3B group and the other two groups $(p<0.05)$. The preoperative location and rotation of L3 was significantly different in bending radiographs of patients in the L3 group with satisfactory and unsatisfactory results.

\section{Discussion}

Controversy exists whether early degenerative changes in the unfused lower lumbar spine following TL/L AIS surgery are related to the number and balance of intervertebral discs of the preserved motion segments. However, most surgeons recommend saving the greatest possible number of lumbar motion segments and maintaining the physiologic sagittal profile $[8,10,18-22]$. This recommendation is based on the high incidence of early degenerative changes in AIS patients treated with fusion at the lowest lumbar levels. The loss of lumbar lordosis after Harrington instrumentation, the so-called flat-back syndrome, can cause early degenerative changes because the remaining distal lumbar motion segments tend to hyperextend in order to compensate for the loss of the more proximal lordosis.

Suk et al. [23] suggested that Zielke ventral derotation system showed more powerful frontal and rotational correction and could be effective even in more rigid or larger curves compared to Cotrel-Dubousset instrumentation (CDI). The CDI system, however, allowed the spine surgeon to easily maintain the physiologic sagittal alignment with the use of a less invasive posterior approach, and had broader indications because it could be extended upwards or downwards to the adjacent curves. Despite these advantages of CDI, it had a limitation in preserving more distal lumbar motion segments because the selection of L4 as the LIV was necessary in order to achieve fusion to the stable vertebra.

Based on our accumulating experience of CDI, we attempted to determine whether the distal fusion level can be the proximal vertebra adjacent to the stable vertebra, or L3 and not L4 in the surgical treatment of the structural thoracolumbar and lumbar curves [14,24]. Lenke et al. [25] suggested stopping the fusion and instrumentation at L3 when: 1) L3 was neutral to grade I rotation, had $<30^{\circ}$ of tilt, and was centered on the sacrum on opposite side-bending; 2) L4 was intersected by the central sacral line with $<20^{\circ}$ of tilt; 3 ) the L3-4 disk was either symmetric or starting to open up toward the convexity of the lumbosacral curve (thus, disk reversal occurred immediately above or below the LIV); and 4) the curve was flexible enough for performing a $90^{\circ}$ rod rotation maneuver using the CDI.

Pedicle screw uses the pedicle, which is mechanically the hardest part of the vertebral body, as an anchor for the fixation devices. Segmental PSI, dispersing the stress on each implant by allowing multiple anchors per spinal level, provides spine surgeons with more powerful correction forces $[16,17,26]$. This enables them to effectively improve deformity correction and to easily restore or maintain the physiologic sagittal alignment. Moreover, segmental PSI offers enhanced three-dimensional correction and preservation of the motion segments by reducing the fusion length, which is comparable to anterior instrumentation.

In our series, none of the patients had thoracolumbar kyphosis or reduced lumbar lordosis in the instrumented zone. All of the patients including those with unsatisfactory results in the L3B group maintained both physiologic thoracic kyphosis and lumbar lordosis without significant loss of sagittal profile.

Few studies have investigated the necessary fusion levels when PSI is used for correction and fixation in AIS surgery. The previous guidelines for determining the level of distal fusion to the stable vertebra in thoracolumbar and lumbar AIS were established during the era of Harrington instrumentation, and they became obsolete with the advent of newer segmental spinal instruments. 
As segmental PSI can derotate and translate the vertebral column three-dimensionally with strong forces, the proximal vertebra adjacent to the stable vertebra before surgery can be put into the stable zone with its horizontalization after surgery. That is, after fusion to the more proximal vertebra than the stable vertebra with segmental PSI, the fused spine can be translated and balanced within the stable zone.

Shufflebarger et al. [14] reported on the thoracolumbar and lumbar curves treated with segmental PSI, and showed that the same levels from the superior to the inferior end vertebrae were fused as those in anterior instrumentation. In spite of the excellent major curve correction of $80 \%$, there were no accurate standard criteria for stopping the distal fusion at L3 with satisfactory results to save the distal lumbar motion segments.

In our opinion, to achieve trunk balance and to minimize early degenerative changes in the unfused distal lumbar motion segments, the fusion mass must be placed within the stable zone with balanced spine and the LIV as horizontal as possible. It was expected that satisfactory results would be obtained whichever type of fusion down to L3 was performed if L3 was placed within the stable zone and had a low tilt after surgery.

As the curve correction using PSI was superior to that observed in the preoperative bending radiograph, it was supposed that L3 would be within the stable zone postoperatively if L3 crossed the mid-sacral line in the bending film towards the concave side (right bending film in the left curve). Because the tilt of the vertebra was associated with three-dimensional rotation deformity of the vertebral body, it was also supposed that the tilt of L3 could be leveled with significant rotational correction if the L3 rotation was less than grade II in the bending film towards the convex side (left bending film in the left curve). We wanted to examine these hypotheses, and therefore, we collected 66 AIS patients in whom the distal fusion level was not determined according to the location and rotation of L3 based on bending films.

In this study, when the preoperative L3 crossed the mid-sacral line with a rotation of less than grade II in both the active bending radiographs, 30 among 33 patients (91.1\%) fused down to L3 showed satisfactory results (Fig. 1). When the preoperative L3 did not cross the mid-sacral line or had rotation of grade II or more in both the bending films, 7 among 8 patients (87.5\%) fused down to L4 showed satisfactory results. Unsatisfactory results were obtained in 15 among 25 patients (68.2\%) in whom L3 did not cross the mid-sacral line or had a rotation of grade II or more when fusion was extended down to L3 (Fig. 2).

The authors have already reported on the selection of the LIV in AIS surgery using PSI $[27,28]$. The results of the current study strongly support our guidelines for selecting the distal fusion level in the treatment of TL/L AIS and provide more data compared to our previous report [29].

Even if there are no clear cut-off values for LIVT and coronal balance that correlate with the clinical outcomes, the authors believe that the definition of unsatisfactory results as a LIVT of more than $10^{\circ}$ or a coronal balance of more than $15 \mathrm{~mm}$ is reasonable to anticipate early degenerative changes in the unfused lumbar spine and to compare among the three groups. Long-term follow-up should be performed to confirm this assumption.

Limitations to this study include its retrospective nature and lack of clinical outcomes. However, the current study suggests the appropriate and exact distal fusion level for surgical correction of TL/L AIS using PSI based on the bending films.

\section{Conclusions}

For the correction of thoracolumbar and lumbar AIS with PSI, the curve can be fused to L3 and not L4; thus, saving one distal motion segment with favorable radiographic outcomes when the preoperative L3 crosses the midsacral line with a rotation of less than grade II in both the active bending radiographs. Otherwise, fusion has to be extended to L4.

\section{Conflict of Interest}

No potential conflict of interest relevant to this article was reported.

\section{References}

1. Lenke LG, Betz RR, Clements D, et al. Curve prevalence of a new classification of operative adolescent idiopathic scoliosis: does classification correlate with treatment? Spine (Phila Pa 1976) 2002;27:604-11.

2. Bunch WH, Chapman RG. Patient preferences in surgery for scoliosis. J Bone Joint Surg Am 1985;67: 
794-9.

3. Dwyer AF, Schafer MF. Anterior approach to scoliosis. Results of treatment in fifty-one cases. J Bone Joint Surg Br 1974;56:218-24.

4. Kaneda K, Fujiya N, Satoh S. Results with Zielke instrumentation for idiopathic thoracolumbar and lumbar scoliosis. Clin Orthop Relat Res 1986;(205):195203.

5. Kaneda K, Shono Y, Satoh S, Abumi K. New anterior instrumentation for the management of thoracolumbar and lumbar scoliosis. Application of the Kaneda two-rod system. Spine (Phila Pa 1976) 1996;21:125061.

6. Hsu LC, Zucherman J, Tang SC, Leong JC. Dwyer instrumentation in the treatment of adolescent idiopathic scoliosis. J Bone Joint Surg Br 1982;64:536-41.

7. Bridwell KH, Betz R, Capelli AM, Huss G, Harvey C. Sagittal plane analysis in idiopathic scoliosis patients treated with Cotrel-Dubousset instrumentation. Spine (Phila Pa 1976) 1990;15:644-9.

8. Cochran T, Irstam L, Nachemson A. Long-term anatomic and functional changes in patients with adolescent idiopathic scoliosis treated by Harrington rod fusion. Spine (Phila Pa 1976) 1983;8:576-84.

9. Debnath UK, Mehdian SM, Webb JK. Spinal deformity correction in duchenne muscular dystrophy: comparing the outcome of two instrumentation techniques. Asian Spine J 2011;5:43-50.

10. Lagrone MO, Bradford DS, Moe JH, Lonstein JE, Winter RB, Ogilvie JW. Treatment of symptomatic flatback after spinal fusion. J Bone Joint Surg Am 1988;70:569-80.

11. Luk KD, Lee FB, Leong JC, Hsu LC. The effect on the lumbosacral spine of long spinal fusion for idiopathic scoliosis. A minimum 10-year follow-up. Spine (Phila Pa 1976) 1987;12:996-1000.

12. Modi HN, Suh SW, Srinivasalu S, Mehta S, Yang JH. Comparison of apical axial derotation between adolescent idiopathic and neuromuscular scoliosis with pedicle screw instrumentation. Asian Spine J 2008;2:74-80.

13. Paonessa KJ, Engler GL. Back pain and disability after Harrington rod fusion to the lumbar spine for scoliosis. Spine (Phila Pa 1976) 1992;17:S249-53.

14. Shufflebarger HL, Geck MJ, Clark CE. The posterior approach for lumbar and thoracolumbar adolescent idiopathic scoliosis: posterior shortening and pedicle screws. Spine (Phila Pa 1976) 2004;29:269-76.

15. Stokes IA. Three-dimensional terminology of spinal deformity. A report presented to the Scoliosis Research Society by the Scoliosis Research Society Working Group on 3-D terminology of spinal deformity. Spine (Phila Pa 1976) 1994;19:236-48.

16. Suk SI, Kim JH, Kim WJ, Kim DS, Lee SM, Kim JH. The influence of segmental pedicle screw fixation on distal fusion level in kingtype I adolescent idiopathic scoliosis. J Korean Soc Spine Surg 1997;4:273-80.

17. Suk SI, Lee CK, Kim WJ, Chung YJ, Park YB. Segmental pedicle screw fixation in the treatment of thoracic idiopathic scoliosis. Spine (Phila Pa 1976) 1995;20:1399-405.

18. Connolly PJ, Von Schroeder HP, Johnson GE, Kostuik JP. Adolescent idiopathic scoliosis. Long-term effect of instrumentation extending to the lumbar spine. J Bone Joint Surg Am 1995;77:1210-6.

19. Edgar MA, Mehta MH. Long-term follow-up of fused and unfused idiopathic scoliosis. J Bone Joint Surg Br 1988;70:712-6.

20. Hayes MA, Tompkins SF, Herndon WA, Gruel CR, Kopta JA, Howard TC. Clinical and radiological evaluation of lumbosacral motion below fusion levels in idiopathic scoliosis. Spine (Phila Pa 1976) 1988;13:1161-7.

21. Large DF, Doig WG, Dickens DR, Torode IP, Cole WG. Surgical treatment of double major scoliosis. Improvement of the lumbar curve after fusion of the thoracic curve. J Bone Joint Surg Br 1991;73:121-4.

22. Thompson JP, Transfeldt EE, Bradford DS, Ogilvie JW, Boachie-Adjei O. Decompensation after CotrelDubousset instrumentation of idiopathic scoliosis. Spine (Phila Pa 1976) 1990;15:927-31.

23. Suk SI, Lee CK, Chung SS. Comparison of zielke ventral derotation system and Cotrel-Dubousset instrumentation in the treatment of idiopathic lumbar and thoracolumbar scoliosis. Spine (Phila Pa 1976) 1994;19:419-29.

24. Monney G, Kaelin AJ. Short posterior fusion for patients with thoracolumbar idiopathic scoliosis. Clin Orthop Relat Res 1999;(364):32-9.

25. Lenke LG, Bridwell KH, Baldus C, Blanke K, Schoenecker PL. Ability of Cotrel-Dubousset instrumentation to preserve distal lumbar motion segments in adolescent idiopathic scoliosis. J Spinal Disord 1993;6:339-50. 
26. Suk SI, Kim WJ, Kim JH, Lee SM. Restoration of thoracic kyphosis in the hypokyphotic spine: a comparison between multiple-hook and segmental pedicle screw fixation in adolescent idiopathic scoliosis. J Spinal Disord 1999;12:489-95.

27. Suk SI. Pedicle screw instrumentation for adolescent idiopathic scoliosis: the insertion technique, the fusion levels and direct vertebral rotation. Clin Orthop
Surg 2011;3:89-100.

28. Suk SI, Kim JH, Kim SS, Lim DJ. Pedicle screw instrumentation in adolescent idiopathic scoliosis. Eur Spine J 2012;21:13-22.

29. Halm H, Niemeyer T, Link T, Liljenqvist U. Segmental pedicle screw instrumentation in idiopathic thoracolumbar and lumbar scoliosis. Eur Spine J 2000;9:191-7. 